\title{
Nanogap Enabled Trajectory Splitting and 3D Optical Coupling in
}

\section{Self-Assembled Microtubular Cavities}

Xiaoyu Wang,,$\uparrow$ Yin Yin,,${ }^{*}$ Haiyun Dong,,${ }^{\dagger}$ Christian N. Saggau $,{ }^{\dagger}, \#, *$ Min Tang, ${ }^{\dagger}$ Lixiang Liu,${ }^{\dagger}$

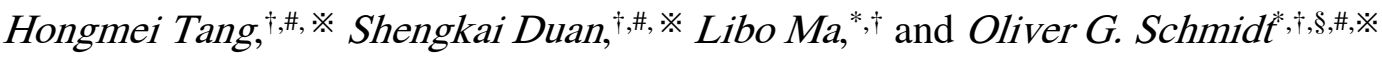

†Institute for Integrative Nanosciences, Leibniz IFW Dresden, 01069 Dresden, Germany

ISchool of Materials Science and Engineering, Jiangsu University, 212013 Zhenjiang, China

\$Faculty of Physics, TU Dresden, 01062 Dresden, Germany

\#Material Systems for Nanoelectronics, TU Chemnitz, 09107 Chemnitz, Germany

*Research Center for Materials, Architectures and Integration of Nanomembranes, TU

Chemnitz, 09126 Chemnitz, Germany 


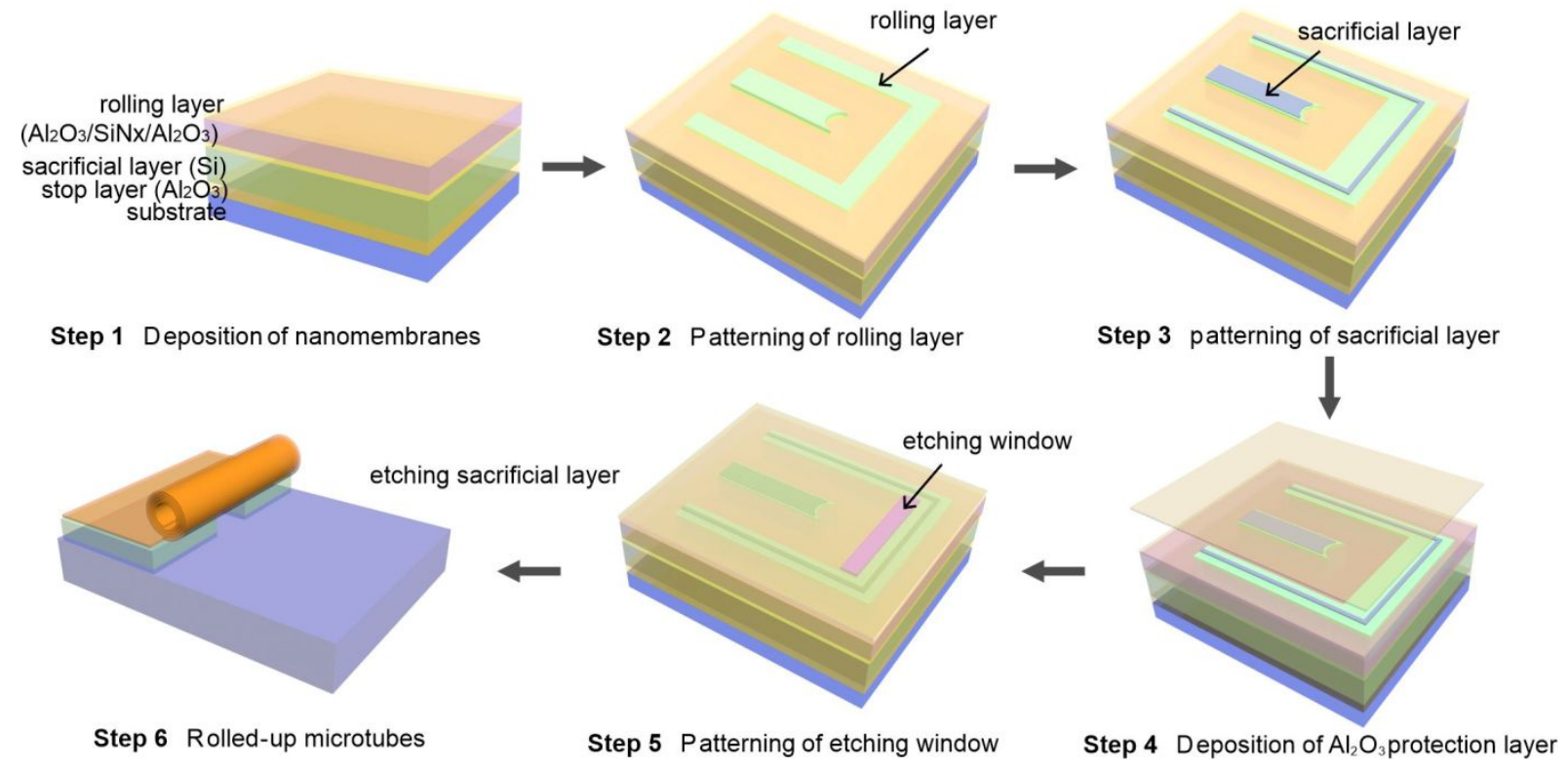

Figure S1. Schematic diagram illustrating the fabrication process steps of the $\operatorname{SiN}_{x}$ microtubular cavities.

First, the rolling layer including a $\mathrm{SiN}_{\mathrm{x}}$ strained nanomembranes and two $\mathrm{Al}_{2} \mathrm{O}_{3}$ protection layers are deposited onto a Si sacrificial layer. A 15-nm-thick $\mathrm{Al}_{2} \mathrm{O}_{3}$ film between the sacrificial layer and substrate was selected as the stop layer to prevent the $\mathrm{Si} / \mathrm{SiO}_{2}$ substrate from being damaged by gas-phase etch. After the deposition of the nanomembrane, photolithography and reactive ion etching (RIE) techniques were used to pattern membrane and sacrificial layer. The patterned sample was passivated by $4 \mathrm{~nm} \mathrm{Al}_{2} \mathrm{O}_{3}$ film. Followed by opening a release window to define the starting rolling edge, the nanomembranes rolled up into a free-standing 3D microtubular cavity by removing the Si sacrificial layer. 


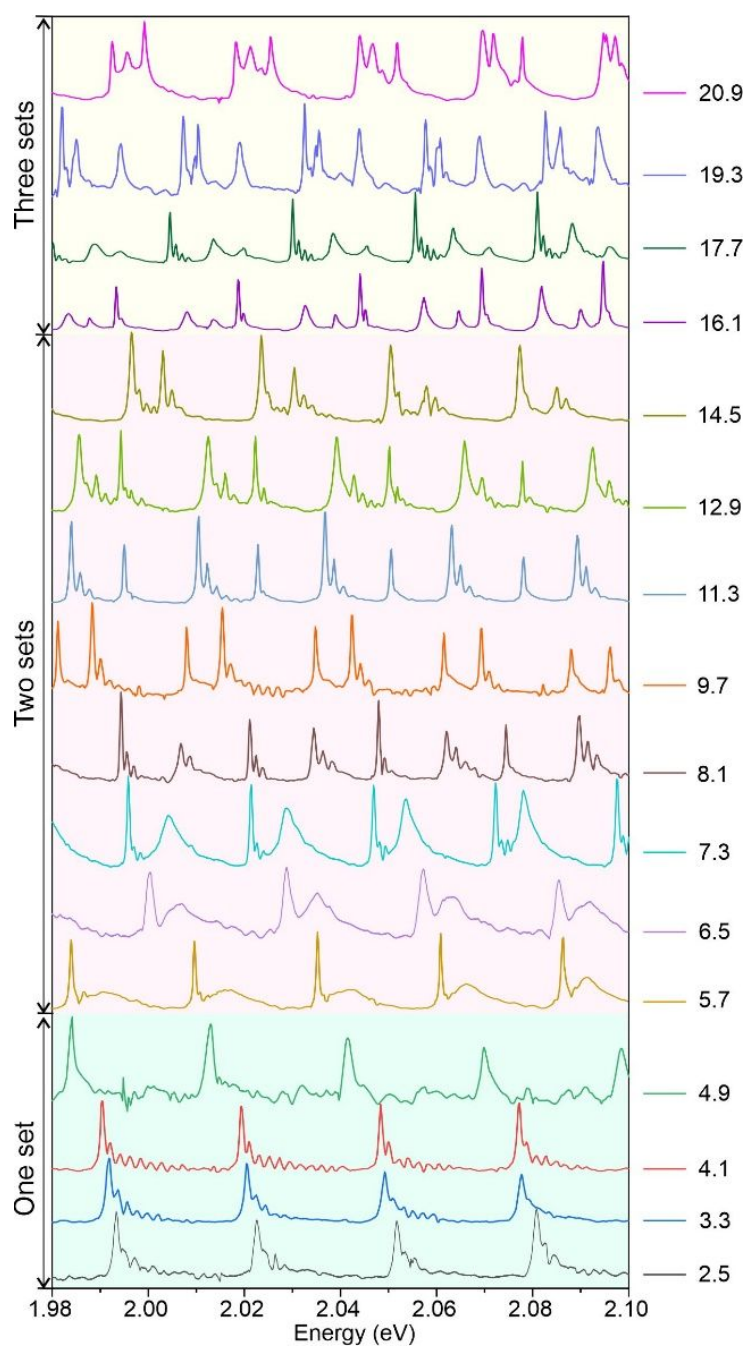

Figure S2. The detailed optical resonant spectra of three types of microtubular cavities with different ratios of rolling lengths to diameter changing from 2.5 to 20.9 and the same rolling step lengths of $30 \mu \mathrm{m}$ per etching cycle. 

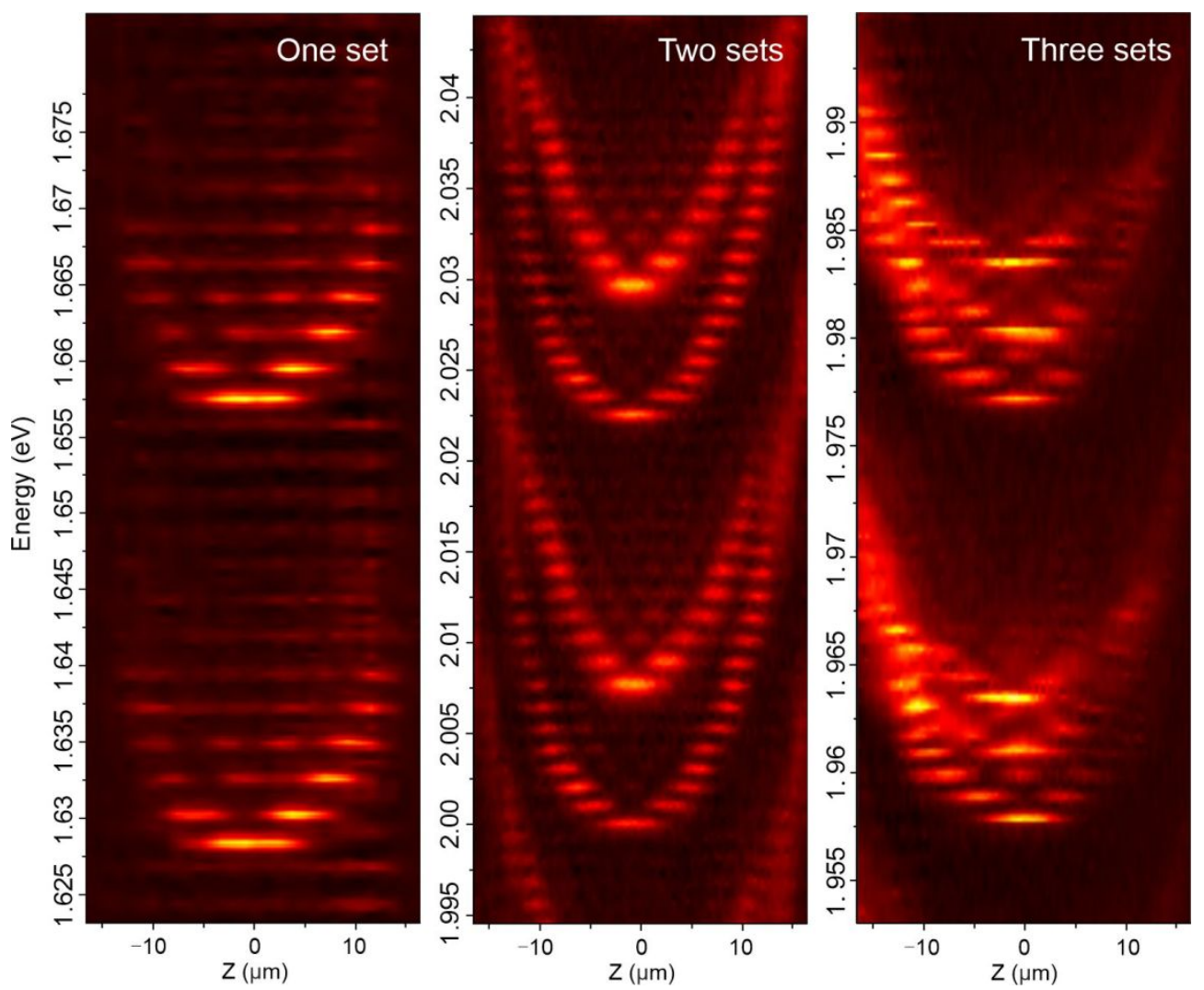

Figure S3. The measured spatially field distributions of axial modes in the lobe region for the three types of microtubular cavities supporting one (left), two (middle), and three (right) sets of resonant modes, respectively. 


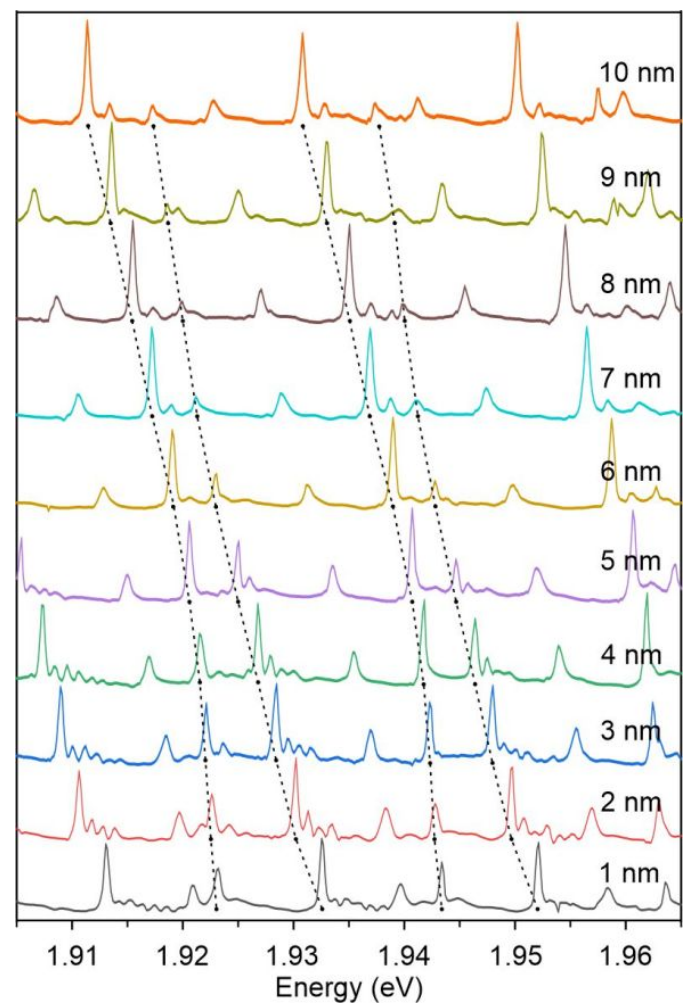

Figure S4 The evolution of measured resonant spectra of the microtubular cavity supporting three sets of resonant modes upon $\mathrm{Al}_{2} \mathrm{O}_{3}$ thickness from 1 to $10 \mathrm{~nm}$ with a step of $1 \mathrm{~nm}$. A clear anticrossing trend is observed with the increase of $\mathrm{Al}_{2} \mathrm{O}_{3}$ thickness. The spectral anticrossing feature together with the modes changing-over are revealed as the direct evidence to verify the strong optical coupling in the single microtubular cavity. 